\title{
Active Phase Conjugation with Multiple Antenna
}

\section{Array Element}

\author{
Pei $\mathrm{Xiao}^{\dagger}$, Senior Member, IEEE, Vincent Fusco ${ }^{\dagger \dagger}$, Fellow IEEE \\ ${ }^{\dagger}$ Centre for Communication Systems Research \\ University of Surrey, Guildford, Surrey, GU2 7XH, UK. \\ E-mail: p.xiaodsurrey.ac.uk \\ ${ }^{\dagger \dagger}$ Institute of ECIT, Queen's University Belfast \\ Belfast, BT3 9DT, UK \\ E-mail: v.fuscodecit.qub.ac.uk
}

\begin{abstract}
In this paper we analyze the temporal and spatial compression characteristics of retrodirective antenna systems wherein active phase conjugation (APC) is deployed. It is shown that under the active phase conjugation Hermitian channel matrix properties result and these lead to opportunities for both temporal and spatial signal compression. The effects of additive channel noise, as well as compression assistance given by multipath richness are described. Some general properties of retrodirective antenna systems with respect to linear and circular polarized pilot signals are also discussed. The scheme elaborated here is compliant with a generic channel model and can therefore be easily incorporated into any readily available communication systems analysis or channel modelling software. The work presented in this paper provides a theoretical foundation for the previous experimental findings in APC systems.
\end{abstract}

\section{INTRODUCTION}

In radio or acoustic underwater communication systems a transmitted signal can follow many different propagation paths before arriving at the receiver. This causes fluctuations in the received signal's amplitude, phase and angle of arrival and as a consequence results in multipath fading. Shallow water acoustic channels [1] and microwave radio channels in office built up environments [2] are particularly problematic as they exhibit a large amounts of multipath interference. Various solutions have been proposed to improve communication speed and reliability in both radio and shallow water acoustic channels. Coherent receivers that use adaptive equalisation have been proposed, e.g., in [3], [4] to 
combat the intersymbol interference (ISI) caused by frequency selective channels. Given accurate channel estimation, ISI can be effectively mitigated by the channel equalisation technique. However, a large number of taps are usually required by the equalisers for dispersive channels and these taps must be constantly adjusted to compensate for the changing environment, resulting in a substantial computational burden.

The phase conjugation technique can be used in order to avoid explicit channel estimation and subsequent equalisation [5]-[8], by implicitly recombing the multipath arrival signals. This technique can be categorized into active phase conjugation (APC) [5], [6] and passive phase conjugation (PPC) [7], [8] approaches.

Antenna arrays are individual radiating elements combined so that they function and perform like a single large antenna. Retrodirective antenna arrays, as the name suggests, have a special feature. When receiving a signal from an unspecified direction, the array can automatically transmit a signal response to that same direction without any previous knowledge of the source direction [6], [9], [10]. This effect is made to occur automatically by sampling the incoming wavefront, and applying the APC technique. Since the phase of the incident signal is now phase conjugated, the signal re-transmitted from the array will self-steer along the direction of the incident wavefront back to the spatial position of the originating pilot tone [11]. The overall system behaves as a directional antenna with omnidirectional coverage and allows tracking of fast moving targets. A related application of this technology is the automotive collision avoidance system which provides the services of warning a driver of potential hazards in his path, including vehicles and roadside obstacles. As illustrated in Fig. 1, vehicle radars first emit modulated continuous waves or pulse waves and receive echoes from other vehicles or roadside obstacles, e.g., man-made traffic structures, topographic structures, etc. Information such as distance and speed can then be determined from the echoes to generate warning signals. Retrodirective arrays need to be placed on the vehicle surfaces and road obstacles for the system to work efficiently. Other applications of retrodirective array include tracking of low-earth orbiting satellites, terrestrial mobile communications, etc.

\section{Active Phase Conjugation Analysis}

Fig. 2 illustrates the principle of APC. The channel impulse response function is $h(t)$ and its Fourier transform is $H(f)$; the transmitted signal is $s(t)$ and its Fourier transform is $S(f)$. The APC scheme operates as follows: the transmitter sends a signal $S(f)$ which traverses the channel $H(f)$, and is observed by the receiver as $S(f) H(f)$; the receiver phase conjugates the signal, producing $S^{*}(f) H^{*}(f)$, and retransmits the signal back to the transmitter. Assuming the channel is quasi-stationary and remains 
static during the return transmission, the signal $S^{*}(f) H^{*}(f)$ travels back through the same channel, resulting in $S^{*}(f)|H(f)|^{2}$ at the transmitter. Consequently, the received signal is a scaled version of the conjugate of the transmitted signal. In this way, it reconcentrates the multipath arrivals and achieves temporal compression.

In contrast with APC, the PPC procedure [8] does not require the return path re-transmission. It achieves temporal compression by cross-correlating two received signals corresponding to the pilot $S_{1}$ and transmitted signal $S_{2}$.

To facilitate analysis, let us use a single antenna APC system and a simple 3-tap sample-spaced discrete channel, shown in Fig. 3, as an example to demonstrate the effectiveness of APC in combatting the multipath effect.

The received signal at the receiver can be expressed as

$$
y_{n}=h_{0} s_{n}+h_{1} s_{n-1}+h_{2} s_{n-2}
$$

where $h_{0}, h_{1}, h_{2}$ are the complex channel gains for the first, second and third taps, respectively, and $s_{n}$ is the transmitted symbol at the $n$th sample instant. The amplitude of each channel coefficient $\left|h_{l}\right|$, $l=0,1,2$ is characterized by a Rayleigh distribution, and the channel gain $\left|h_{l}\right|^{2}$ follows a Chi-square distribution with 2 degrees of freedom. The total average channel gain $\sum_{l=0}^{2} E\left[\left|h_{l}\right|^{2}\right]$ is normalized to unity.

After phase conjugation and return path re-transmission the signal received at the transmitter side after a round-trip through the example 3-tap system is

$$
\begin{aligned}
z_{n} & =h_{0} y_{n-2}^{*}+h_{1} y_{n-1}^{*}+h_{2} y_{n}^{*} \\
& =R(0) s_{n-2}^{*}+R(1) s_{n-1}^{*}+R^{*}(1) s_{n-3}^{*}+R(2) s_{n}^{*}+R^{*}(2) s_{n-4}^{*},
\end{aligned}
$$

where $R(\tau)$ is the instantaneous correlation function (ICF) of the channel at the $\tau$ th lag, and it depends on the instantaneous value of channel coefficients, i.e.,

$$
R(\tau)=h_{i} h_{i-\tau}^{*}
$$

In the case of 3-tap channel,

$$
\begin{aligned}
& R(0)=\left|h_{0}\right|^{2}+\left|h_{1}\right|^{2}+\left|h_{2}\right|^{2} ; \\
& R(1)=h_{1} h_{0}^{*}+h_{2} h_{1}^{*} ; \\
& R(2)=h_{2} h_{0}^{*} .
\end{aligned}
$$

Note that in the above derivation, the channel is assumed to be quasi-stationary such that the channel gains remain static during the forward transmission and return re-transmission. 
Eq. (1) corresponds to the forward transmission process illustrated in upper part of Fig. 2, whereas Eq. (2) corresponds to the return path re-transmission illustrated in the lower part of Fig. 2. These two equations show the time-domain convolution process between the channel and transmitted signal. According to the Fourier theorem, the time-domain convolution and time-reversal, shown by Eqs. (1) and (2), correponds to the frequency-domain multiplication and conjugation illustrated in Fig. 2.

From Eqs. (2), (3), it can be seen that the principle of APC operation is that it coherently reconcentrates the multipath arrivals at zero-th time lag (temporal compression). A special example is a single tap channel which results in a channel ICF with a strong peak at the zero-th lag and zero for all the other lags.

For an $L$-tap channel, Eq. (2) can be generalized to

$$
z_{n}=R(0) s_{n-L+1}^{*}+R(1) s_{n-L+2}^{*}+R^{*}(1) s_{n-L}^{*}+R(2) s_{n-L+3}^{*}+R^{*}(2) s_{n-L-1}^{*}+\ldots
$$

It should be noted that these ICFs may also have temporal sidelobes that result in residual intersymbol interference. It is therefore desirable to reduce the sidelobes of the channel ICFs. Table I shows simulation results based on the IEEE 802.11 WLAN channel model [12].

The results for the number of array elements equal to 1 and 10 indicate that the 51-tap channel exhibits better correlation properties with reduced sidelobes. A rich scattering environment will reduce intersymbol interference through temporal compression because in this case, the channel coefficients appear noise-like, resulting in an ICF that has a strong peak at the zero-th lag and is nearly zero at all other lags. Thus an APC system operating in a multipath rich environment is expected to exhibit better intersymbol interference mitigation properties than as operating in a multipath poor environment.

\section{AnAlysis of Multi-Antenna Array APC System}

In this section, we analyze the APC system with antenna array. An antenna array is a set of two or more antennas, from which the signals are combined or processed in order to achieve improved performance over that of a single antenna. It is obvious to see from Table I that an antenna array positioned at the receiver facilitates better temporal compression since the main peaks from different elements are added coherently, whereas sidelobes are spread out due to incoherent summation of the sidelobes from different elements.

Suppose we have an $N_{r}$-element antenna array at receiver, antenna correlation factor [13] between the $i$-th and $m$-th element is defined as

$$
\rho_{i, m}=\left|\frac{\mathrm{E}\left\{\left(h_{i}-E\left\{h_{i}\right\}\right)\left(h_{m}-E\left\{h_{m}\right\}\right)^{*}\right\}}{\sqrt{\mathrm{E}\left\{\left|h_{i}-E\left\{h_{i}\right\}\right|^{2}\right\} \mathrm{E}\left\{\left|h_{m}-E\left\{h_{m}\right\}\right|^{2}\right\}}}\right|,
$$


where $h_{i}$ and $h_{m}$ are the channel coefficients between the transmit antenna and the $i$-th and $m$-th element of the receive antenna array, respectively. By assuming that the antenna correlation factor is identical for all elements in the array irrespective of their positions, i.e., $\rho_{i, m}=\rho$ for $i=1, \ldots, N_{r} ; m=$ $1, \ldots, N_{r} ; i \neq m$, we examine the impact of antenna correlations on channel ICF in Table II. Here the slidelobes of the channel ICF become larger as the correlation factor $\rho$ increases. Thus antenna correlations adversely affect the ICF of the channel and diminish sidelobe averaging effects.

In the multi-antenna array APC system shown in Fig. 4, the transmitter has $2 \times 1$ array elements and the receiver has $M \times N$ array elements. An array of $M \times N$ elements is defined as a spatially extended collection of $M \times N$ similar radiators, having the same polar radiation patterns and the same orientation in 3D space. The system has 2 pilot sources which transmit pilots $S_{1}$ and $S_{2}$ from the $2 \times 1$ array elements to which the $M \times N$ phase conjugate array will self steer the return signal to. These pilot signals can be transmitted with either vertical (horizontal) linear polarisation or circular polarisation depending on the application, e.g., linear polarisation for terrestrial mobile communications, and circular polarisation for ground to satellite communications. The task is to obtain the signals at their originating positions after a round trip through the system. The multipath channel contains direct and indirect paths due to scatterers. In the channel the signal experiences time delay and phase shift as well as attenuation of amplitude due to free-space path loss. The received signals are locally phase conjugated and re-transmitted through the same element. We assume the channel has rich scattering properties, thus based on our findings in Section II, intersymbol interference effects can be neglected. The received signal at $N_{r}=M \times N$ array elements can be approximated as

$$
\underbrace{\left[\begin{array}{c}
Y_{1} \\
Y_{2} \\
\vdots \\
Y_{N_{r}}
\end{array}\right]}_{\mathbf{Y}} \approx \underbrace{\left[\begin{array}{cc}
H_{11} & H_{12} \\
H_{21} & H_{22} \\
\vdots & \vdots \\
H_{N_{r} 1} & H_{N_{r} 2}
\end{array}\right]}_{\mathbf{H}} \underbrace{\left[\begin{array}{c}
S_{1} \\
S_{2}
\end{array}\right]}_{\mathbf{S}}+\underbrace{\left[\begin{array}{c}
V_{1} \\
V_{2} \\
\vdots \\
V_{N_{r}}
\end{array}\right]}_{\mathbf{V}},
$$

where $H_{i j}$ is the channel coefficient between the $i$-th receive antenna array element and the $j$-th transmmit antenna array element; $V_{i}$ stands for the noise at the $i$ th array element $\left(i=1, \ldots, N_{r}\right)$, it is assumed to be a complex Gaussian random variable with zero mean and variance $\sigma_{v}^{2}$, i.e., $V_{i} \sim$ $\mathcal{C N}\left(0, \sigma_{v}^{2}\right)$. Note that the channel matrix $\mathbf{H}$ in Eq. (5) would be a block toeplitz matrix in presence of intersymbol interference [14].

The signal received at the transmitter side after a round trip through the system shown in Fig. 2 is 
$\mathbf{Z}=\mathbf{H}^{\mathcal{T}} \mathbf{Y}^{*}$, (where $\mathbf{H}^{\mathcal{T}}$ is the transposed version of $\mathbf{H}$ ), i.e.,

$$
\begin{aligned}
\underbrace{\left[\begin{array}{l}
Z_{1} \\
Z_{2}
\end{array}\right]}_{\mathbf{Z}}= & \underbrace{\left[\begin{array}{llll}
H_{11} & H_{21} & \ldots & H_{N_{r} 1} \\
H_{12} & H_{22} & \ldots & H_{N_{r} 2}
\end{array}\right]}_{\mathbf{H}^{\mathcal{T}}}(\underbrace{\left[\begin{array}{cc}
H_{11}^{*} & H_{12}^{*} \\
H_{21}^{*} & H_{22}^{*} \\
\vdots & \vdots \\
H_{N_{r} 1}^{*} & H_{N_{r} 2}^{*}
\end{array}\right]\left[\begin{array}{c}
S_{1}^{*} \\
S_{2}^{*}
\end{array}\right]+\left[\begin{array}{c}
V_{1}^{*} \\
V_{2}^{*} \\
\vdots \\
V_{N_{r}}^{*}
\end{array}\right]}) \\
= & \underbrace{\left[\begin{array}{ll}
\sum_{i=1}^{N_{r}}\left|H_{i 1}\right|^{2} & \sum_{i=1}^{N_{r}} H_{i 1} H_{i 2}^{*} \\
\sum_{i=1}^{N_{r}} H_{i 1}^{*} H_{i 2} & \sum_{i=1}^{N_{r}}\left|H_{i 2}\right|^{2}
\end{array}\right]}_{\mathbf{R}}\left[\begin{array}{c}
S_{1}^{*} \\
S_{2}^{*}
\end{array}\right]+\left[\begin{array}{l}
\sum_{i=1}^{N_{r}} H_{i 1} V_{i}^{*} \\
\sum_{i=1}^{N_{r}} H_{i 2} V_{i}^{*}
\end{array}\right] .
\end{aligned}
$$

The matrix $\mathbf{R}$ is formed as $\mathbf{R}=\mathbf{H}^{\mathcal{T}} \mathbf{H}^{*}$, its off-diagonal elements are

$$
\begin{aligned}
& R_{12}=\sum_{i=1}^{N_{r}} H_{i 1} H_{i 2}^{*} \\
& R_{21}=\sum_{i=1}^{N_{r}} H_{i 1}^{*} H_{i 2} .
\end{aligned}
$$

Obviously, $\mathbf{R}$ is a Hermitian matrix since $R_{i j}=R_{j i}^{*}$. In order to achieve transmit diversity ${ }^{1}$, we transmit the same pilot signal from the transmit antenna array elements (i.e., $S_{1}=S_{2}=S$, a scenario of linear polarization), then combine the returned signals at transmitter, resulting in

$$
Z=Z_{1}+Z_{2}=\left[\sum_{i=1}^{N_{r}}\left(\left|H_{i 1}\right|^{2}+\left|H_{i 2}\right|^{2}\right)+2 \operatorname{Re}\left\{\sum_{i=1}^{N_{r}} H_{i 1} H_{i 2}^{*}\right\}\right] S^{*}+\sum_{i=1}^{N_{r}}\left(H_{i 1}+H_{i 2}\right) V_{i}^{*} .
$$

Eq. (7) shows that each frequency component of the return signal $Z$ is a scaled and conjugate version of the originally transmitted signal $S$ (corrupted with additive noise), which is true regardless of antenna polarization. This follows the fact that for a Hermitian matrix, addition of its off-diagonal elements results in a real-valued scaling factor, no signal rotations are incurred. It is also obvious to see from Eq. (7) that the retrodirective array has the capability of redirecting energy back along incoming multipaths such that they will coherently combine at the original source location. This proves our previous experimental finding reported in [10].

Eqs. (6) and (7) also hold for APC systems with antenna correlations (mutual couplings) since antenna correlations do not change the fact that the matrix $\mathbf{R}$ is Hermitian. However, antenna correlations do

\footnotetext{
${ }^{1}$ Transmit diversity is radio communication technique using signals that originate from two or more independent sources that have been modulated with identical information-bearing signals and that may vary in their transmission characteristics at any given instant. It can help overcome the effects of fading, outages, and circuit failures.
} 
affect the ICF of the channel and act to diminish the sidelobe averaging effects as discussed in the previous section. Therefore, it is desirable to design systems with low antenna correlations.

Next we derive expressions of the received signal for the following special scenarios:

i) Assume $N_{r}=M \times N=2$ and that the transmitter has only one antenna, $S_{1}=S_{2}=S$ (linear polarization), we obtain

$$
Z=Z_{1}+Z_{2}=\left[\sum_{i=1}^{2}\left(\left|H_{i 1}\right|^{2}+\left|H_{i 2}\right|^{2}\right)+2 \operatorname{Re}\left\{\sum_{i=1}^{2} H_{i 1} H_{i 2}^{*}\right\}\right] S^{*}+\sum_{i=1}^{2}\left(H_{i 1}+H_{i 2}\right) V_{i}^{*} .
$$

Since $H_{11}=H_{12}=H_{1}$ and $H_{21}=H_{22}=H_{2}$ thus

$$
Z=2\left[\left|H_{1}\right|^{2}+\left|H_{2}\right|^{2}\right] S^{*}+2\left[H_{1} V_{1}^{*}+H_{2} V_{2}^{*}\right]
$$

The received signal is a scaled and conjugated version of the transmitted signal plus additive noise.

(ii) If $S_{1}=S e^{j \alpha}$ is oriented along the horizontal axis and $S_{2}=S e^{j \alpha \pm \frac{\pi}{2}}$ is oriented along the vertical axis ( $\alpha$ is an arbitrary phase shift), then from Eq. (6) we conclude that a transmitted circularly polarized signal will be received with the same hand of polarization but with its phase conjugated.

\section{Spatial Reception Properties}

Now we investigate the scenario when a signal after a round trip transmission through the system is received not only by the two antennas that originally transmit pilots $S_{1}$ and $S_{2}$, but also by two other antennas located at different spatial positions. This scenario arises when the special focusing properties of the APC arrangement are to be evaluated. By using different receive antennas at different spatial locations we provide a means by which we can sample the region surrounding the antennas that originally transmit pilots. In this case, Eq. (6) can be reformed as

$$
\mathbf{Z}=\left[\begin{array}{l}
\mathbf{H}^{\mathcal{T}} \\
\mathbf{G}^{\mathcal{T}}
\end{array}\right] \mathbf{Y}^{*}=\left[\begin{array}{l}
\mathbf{H}^{\mathcal{T}} \mathbf{Y}^{*} \\
\mathbf{G}^{\mathcal{T}} \mathbf{Y}^{*}
\end{array}\right]=\left[\begin{array}{l}
\mathbf{H}^{\mathcal{T}} \mathbf{H}^{*} \mathbf{S}^{*}+\mathbf{H}^{\mathcal{T}} \mathbf{V}^{*} \\
\mathbf{G}^{\mathcal{T}} \mathbf{H}^{*} \mathbf{S}^{*}+\mathbf{G}^{\mathcal{T}} \mathbf{V}^{*}
\end{array}\right]
$$

where the vector $\mathbf{Z}$ is re-defined as $\left[\begin{array}{llll}Z_{1} & Z_{2} & Z_{3} & Z_{4}\end{array}\right]^{\mathcal{T}} ; G_{i j}$ is the channel coefficient between the $j$ th $(j=3,4)$ antenna at the transmitter side and the $i$ th antenna at the receiver side, and

$$
\mathbf{G}^{\mathcal{T}}=\left[\begin{array}{cccc}
G_{13} & G_{23} & \ldots & G_{N_{r} 3} \\
G_{14} & G_{24} & \ldots & G_{N_{r} 4}
\end{array}\right] ; \quad \mathbf{Y}^{*}=\underbrace{\left[\begin{array}{ccc}
H_{11}^{*} & H_{12}^{*} \\
H_{21}^{*} & H_{22}^{*} \\
\vdots & \vdots \\
H_{N_{r} 1}^{*} & H_{N_{r} 2}^{*}
\end{array}\right]}_{\mathbf{H}^{*}} \underbrace{\left[\begin{array}{c}
S_{1}^{*} \\
S_{2}^{*}
\end{array}\right]}_{\mathbf{S}^{*}}+\underbrace{\left[\begin{array}{c}
V_{1}^{*} \\
V_{2}^{*} \\
\vdots \\
V_{N_{r}}^{*}
\end{array}\right]}_{\mathbf{V}^{*}}
$$

In Eq. (10), $\mathbf{R}=\mathbf{H}^{\mathcal{T}} \mathbf{H}^{*}$ is a Hermitian matrix, whereas this is not true for $\mathbf{G}^{\mathcal{T}} \mathbf{H}^{*}$. The conclusion is that the signal received at the first two antennas that transmit $S_{1}$ and $S_{2}$ exhibit the special compression 
properties discussed earlier. However for signals received at the other two antennas at different spatial locations, effective high quality compression will not occur. Consequently, at positions more remote from the original source positions, recovery of data becomes increasingly more difficult, this was demonstrated experimentally in [10].

\section{Simulation Results}

Simulation results are shown in this section for the system analyzed in Section III. The system has 2 pilot sources transmitting pilots $S_{1}$ and $S_{2}$, which are 90 degree phase rotated version of each other, and each represents a block of 200 QPSK symbols. These symbol sets are transmitted over 11-path 802.11 WLAN channels and the results are averaged over three thousand channel and noise realizations.

The effect of additive noise is examined in Fig. 5, where we see the performance of APC as a function of SNR defined by $E_{b} / N_{0}$ in $\mathrm{dB}$ scale, the number of array elements is set to be $N=20$. A 20-element phase conjugating array represents a typical scenario that could be realised from a practical perspective, such that a well collimated return signal to the pilot tone location will occur. If more sources (pilots at the same frequency) are added, their effect is simply to cause the signal returned from the phase conjugating array to be shared out to each of the pilot tone locations. Since these systems are designed to provide high precision retrodirective action on a single carrier we have selected two sources, nominally collocated, in order to investigate the main properties of the system for single carrier tracking under different polarisation states.

The following seven different receiver schemes have been tested:

- Scheme 1: only $Z_{1}$ in Eq. (6) is used to decode the transmitted symbols;

- Scheme 2: only $Z_{2}$ in Eq. (6) is used to decode the transmitted symbols;

- Scheme 3: only $Z_{3}$ in Eq. (10) is used to decode the transmitted symbols;

- Scheme 4: only $Z_{4}$ in Eq. (10) is used to decode the transmitted symbols;

- Scheme 5: Both $Z_{1}$ and $Z_{2}$ are used to decode the transmitted symbols. Since the 90 degree phase shift between $S_{1}$ and $S_{2}$, the received signal is combined such that $Z=Z_{1}-j Z_{2}$.

- Scheme 6: $Z_{1}, Z_{2}, Z_{3}, Z_{4}$ are all used to decode the transmitted symbols, the signal is combined such that $Z=Z_{1}-j Z_{2}+Z_{3}-j Z_{4}$.

- Scheme 7: $Z_{3}, Z_{4}$ are used to decode the transmitted symbols, the signal is combined such that $Z=Z_{3}-j Z_{4}$.

One can see from Fig. 5 that Scheme 5 achieves the best performance compared to all the other schemes due to the diversity gain obtained by combining $Z_{1}$ and $Z_{2}$. The APC system cannot operate with Schemes 3 and 4 because $\mathbf{G}^{\mathcal{T}} \mathbf{H}^{*}$ in Eq. (10) is no longer Hermitian, and consequently the compression 
effect is not guaranteed. On the contrary, the use of $Z_{3}$ and $Z_{4}$ serves as an additional source of noise due to the randomness of $\mathrm{G}^{\mathcal{T}} \mathbf{H}^{*}$, thus degrades system performance. Scheme 6 exploits the diversity gain by coherently combining $Z_{1}$ and $Z_{2}$, however, it also suffers from additional noise due to $Z_{3}$ and $Z_{4}$. Therefore, it exhibits a worse performance than Scheme 5. Schemes 1 and 2 have almost the identical performance, but both are inferior to Scheme 5 due to the lack of diversity gain. In all the investigated scenarios, noise has an adverse effect on the system performance, thus a reasonable level of SNR needs to be maintained for the proper functioning of APC. For example, if an APC system under Scheme 5 has a target bit error rate of $10^{-3}$, it has to operate in an environment where the SNR has to be greater than $8.4 \mathrm{~dB}$.

In Fig. 6, we show the performance of APC as a function of the number of array elements $N_{r}$ for the various schemes mentioned above. Here the SNR value is fixed at $E_{b} / N_{0}=12 \mathrm{~dB}$. For Schemes $1,2,5,6$, the system performance improves as the number of array elements increases, especially for Scheme 5 with diversity exploited in the decoding process. As analyzed earlier, better correlation property and thus better compression can be achieved by increasing $N_{r}$, leading to better BER performance, as verifed in Fig. 6.

In Fig. 7, we show the performance of APC as a function of SNR for different values of $N_{r}$ under Scheme 5. The employed 11-path WLAN channel is by itself sufficiently rich in scattering such that the BER floor at high SNR does not occur even when a very small number of array elements are employed at receiver, e.g., when $N_{r}=5$. The most noticeable gain is observed when increasing $N_{r}$ from 5 to 10, after which point performance gain gradually becomes saturated as the number of array elements is further increased.

In Fig. 8, we show the performance of APC as a function of the number of array elements $N_{r}$ for different levels of SNR under Scheme 5. In harsh channels with high noise level, e.g., when $E_{b} / N_{0}=$ $4 \mathrm{~dB}$, the system performance cannot be significantly improved by inceasing $N_{r}$ since noise is the dominant factor. The gain achieved by increasing $N_{r}$ is more obvious at high SNRs, e.g., when $E_{b} / N_{0}=$ $13 \mathrm{~dB}$, since noise becomes less dominant, and intersymbol interference plays the more important role in system performance. The SNR level needs to be reasonably large in order for the multi-array APC system to be beneficial. In this case, a larger number of array elements will achieve better compression, thus less intersymbol interference and better BER performance.

In Fig. 9, we examine the impact of antenna correlations on the performance of APC. Here we assume the correlation factor $\rho$ (a scalar between 0 and 1) is identical for all antennas in the array irrespective of their positions. Fig. 9 indicates that the performance of the APC system with Schemes 1 and 5 becomes worse as the channels become more correlated ( $\rho$ increases). As analyzed in Section I, antenna 
correlations adversely affect the ICF of the channel and diminish sidelobe averaging effects. However, when decoded with $Z_{3}$ (Scheme 3), the system performance improves marginally as $\rho$ increases. This follows from the fact that when the $\mathbf{G}$ matrix in Eq. (10) is more correlated to the $\mathbf{H}$ matrix as is the case when $\rho$ increases, $\mathbf{G}^{\mathcal{T}} \mathbf{H}^{*}$ starts to resemble a Hermitian matrix, thus the compression effect is achieved to a certain degree. This effect is more obvious when both $Z_{3}$ and $Z_{4}$ are used for symbol decoding (Scheme 7) as indicated by the figure, again reinforcing our earlier comments on spatial and temporal compression.

\section{CONCLUSIONS}

The temporal and spatial compression characteristics of retrodirective antenna systems with multiple antenna array elements have been discussed in this paper. It has been shown by using a discrete time channel transfer function model that under the active phase conjugation Hermitian channel matrix properties result and that these lead to opportunities for both temporal and spatial signal compression. Simulations based on QPSK transmission in conjunction with an 802.11 wireless propagation model are used to demonstrate these effects. The theoretical framework developed in this paper enables us to verify some properties of active phase conjugation systems with respect to linearly and circularly polarized signals. For example, a transmitted linearly/circularly polarized signal will be received with the same kind of polarization but with its phase conjugated.

\section{ACKNOWLEDGEMENT}

The work was carried out under the support of the UK Engineering and Physical Science Research Council (EPSRC) under grant EP01707X/1 and the Northern Ireland Department of Education and Learning Strengthening All Island Scheme, Mobile Wireless Futures Programme.

\section{REFERENCES}

[1] T. Yi, X. Xu. "Simulation study of multi-path characteristics of acoustic propagation in shallow water wireless channel", International Conference on Wireless Communication, Networking and Mobile Computing, pp. 1068-1070, 2007.

[2] P. Boyer. "Performance based on selective multipath reception", IEEE Trans. Commun., vol. 52, no. 2, pp. 280-288, 2004.

[3] M. Stojanovic, J. Catipovic, J. Proakis. "Phase-coherent digital communications for underwater acoustic channels". IEEE Journal of Oceanic Engineering, vol. 19, pp. 100-111, January 1994.

[4] M. Stojanovic. "Recent advances in high-speed underwater acoustic communication". IEEE Journal of Oceanic Engineering, vol. 21, pp. 125-136, April 1996.

[5] D. Jackson, D. Dowling. "Phase-conjugation in underwater acoustics", J. Acoust. Soc. Am., vol 95, no.3, pp. 1450-1458, 1991.

[6] B. Y. Toh, V. F. Fusco, N. B. Buchanan. "Assessment of performance limitations of PON retrodirective arrays", IEEE Trans. antennas and propagation, vol. 50, no. 10, pp. 1425-1432, 2002. 
[7] D. Rouseff, D. Jackson, W. Fox, C. Jones, J. Ritcey, D. Dowling. "Underwater acoustic communication by passive-phase conjugation: theory and experimental results". IEEE Journal of Oceanic Engineering, vol. 26, no. 4, pp. 821-831, 2001.

[8] P. Hursky, M. Porter, M. Siderius. "Point-to-point underwater acoustic communications using spread-spectrum passive phase conjugate", Journal of the Acoustical Society of America, vol. 120, no. 1, pp. 247-257, July 2006.

[9] K. Leong, R. Miyamoto, T. Itoh. "Moving forward in retrodirective antenna array". IEEE Potentials, vol. 22, no. 3, pp. 16-21, August 2003.

[10] N. B. Buchanan, V. F. Fusco. "Bit error rate performance enhancement of a retrodirective array over a conventional fixed beam array in a dynamic multipath environment”, IEEE Trans. antennas and propagation, vol. 58, no. 4, pp. 757-762, April 2010.

[11] C. Y. Pon. "Retrodirective array using the heterodyne technqiue", IEEE Trans. antennas and propagation, vol. AP-12, no. 2, pp. 176-180, March 1964.

[12] B. O’Hara, A. Petrick. IEEE 802.11 Handbook: A Designer's Companion. 2nd Edition, IEEE, 2005.

[13] M. K. Ozdemir, E. Arvas. "Dynamics of spatial correlation and implications on MIMO systems". IEEE MTT-S International Microwave Symposium, pp. 1723-1726, 2005.

[14] L. Barbero, P. Xiao, T. Ratnarahah, M. Sellathurai, C. Cowan. "A sphere decoder with approximate QR decomposition for frequencyselective channels". Proc. IEEE International Conference on Communications, May 2010. 


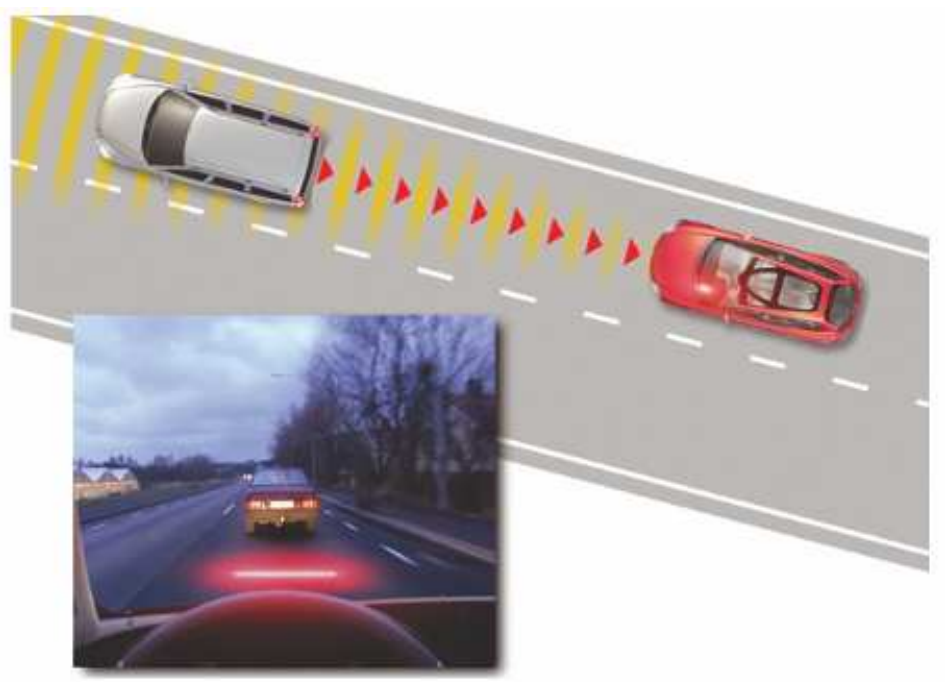

Fig. 1. Illustration of an automotive collision avoidance system which warns the driver a potential collision. The radar system installed in the red car first sends an interrogating signal (an interrogating signal is used by an electronic device to detect the presence of surrounding objects, to estimate their distances and moving speeds) indicated by the yellow beams, the retrodirective array mounted on the gray car upon receiving the signal, applies the principle of APC, i.e., phase conjugates the signal, and retransmits this signal back to the red car to track the gray car's distance, speed, etc. and generate warning if necessary. When the warning signal is generated, the driver will see the flashing lights reflected in the windshield. 


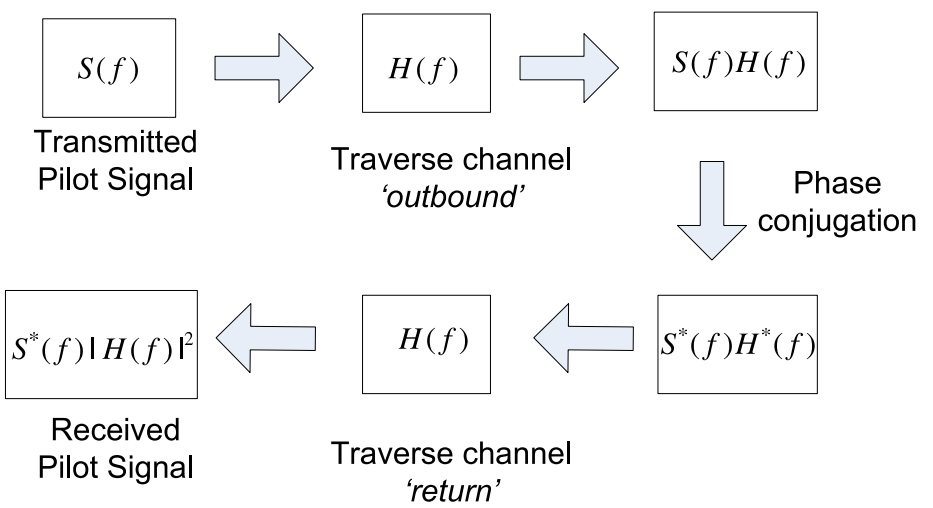

Fig. 2. Active Phase Conjugation. 


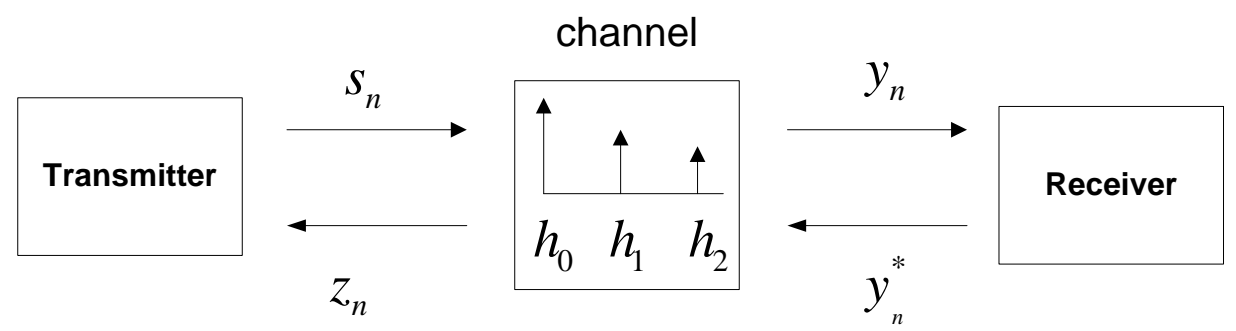

Fig. 3. Discrete time model for single antenna array APC system. 


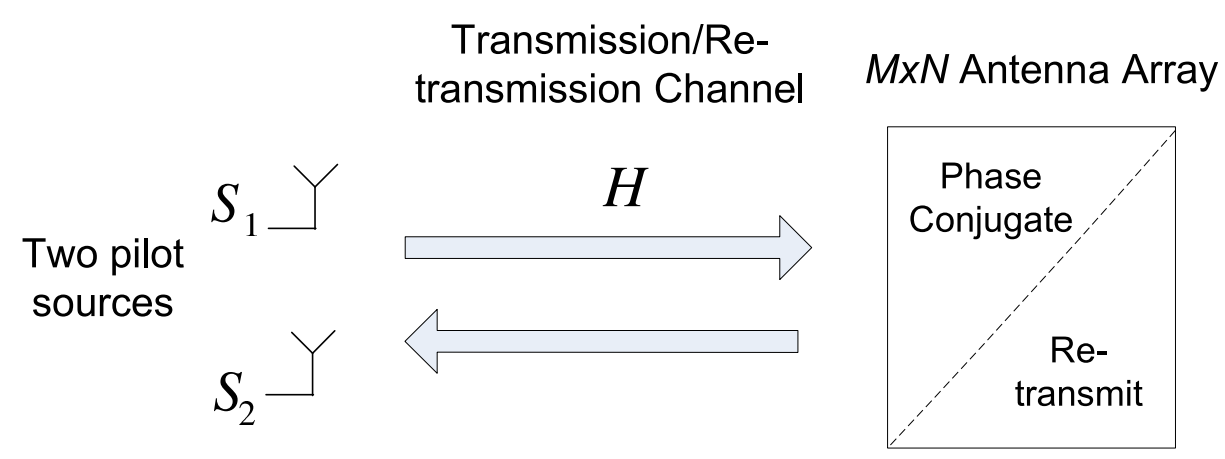

Fig. 4. Multi-antenna array APC system under investigation. The pilot sources are the transmit array elements which are used to transmit pilot signals. 


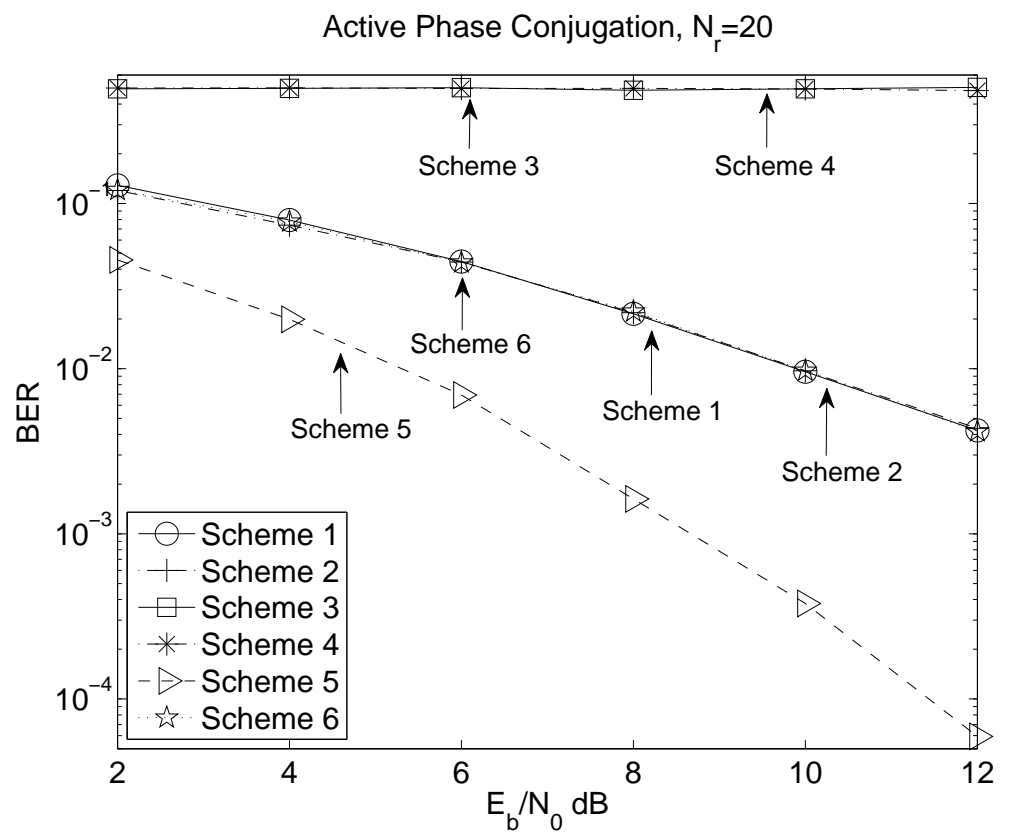

Fig. 5. Performance of APC as a function of SNR for 11-tap WLAN channel, $N_{r}=20$. 


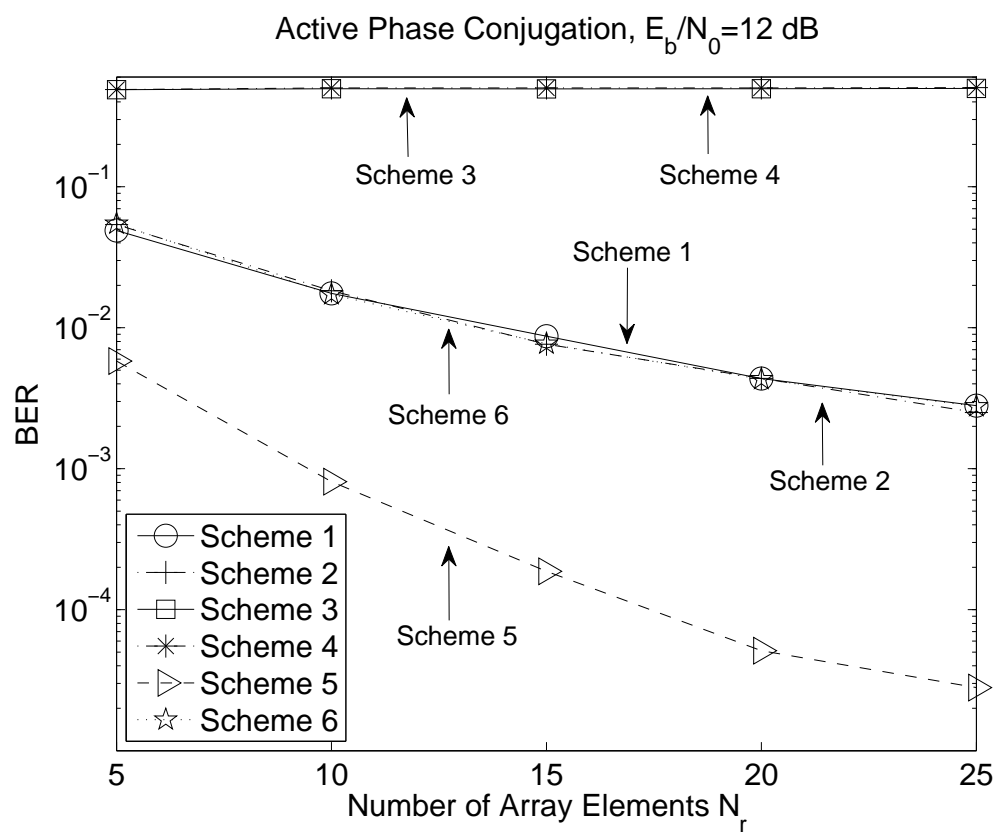

Fig. 6. Performance of APC as a function of $N_{r}$ for 11-tap WLAN channel. 


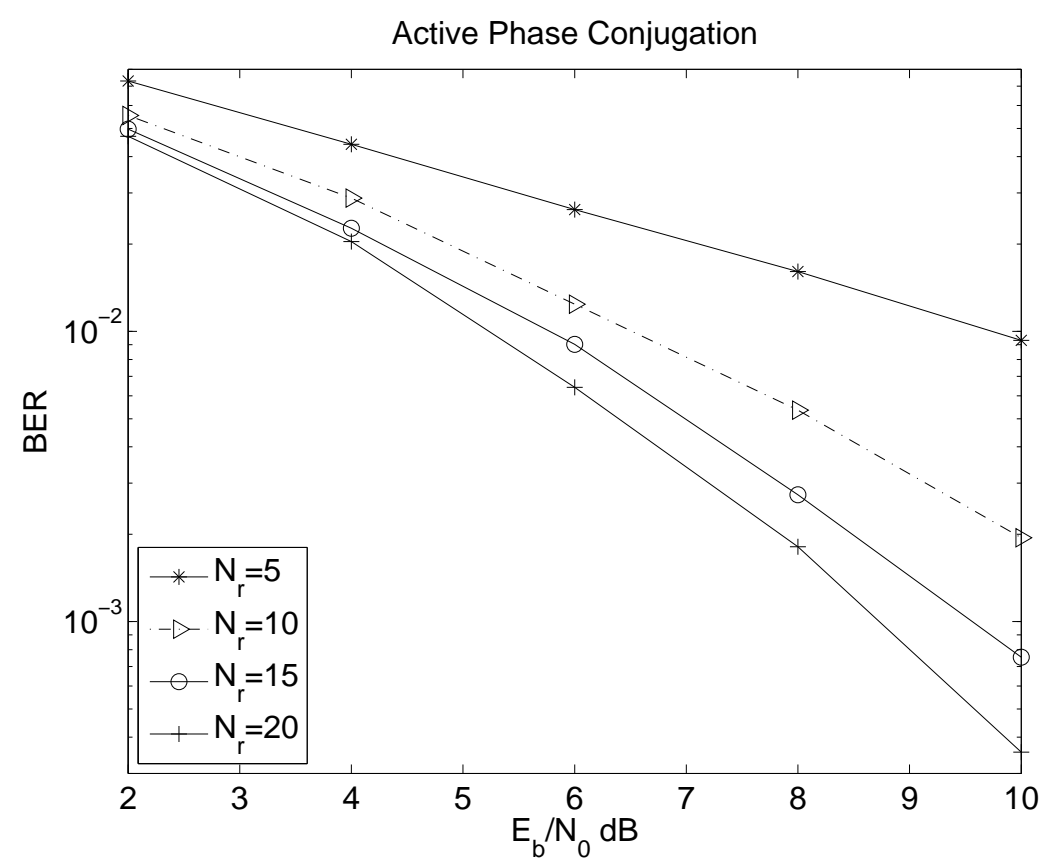

Fig. 7. Performance of APC (Scheme 5) as a function of SNR for 11-tap WLAN channel, $N_{r}=5,10,15,20$. 


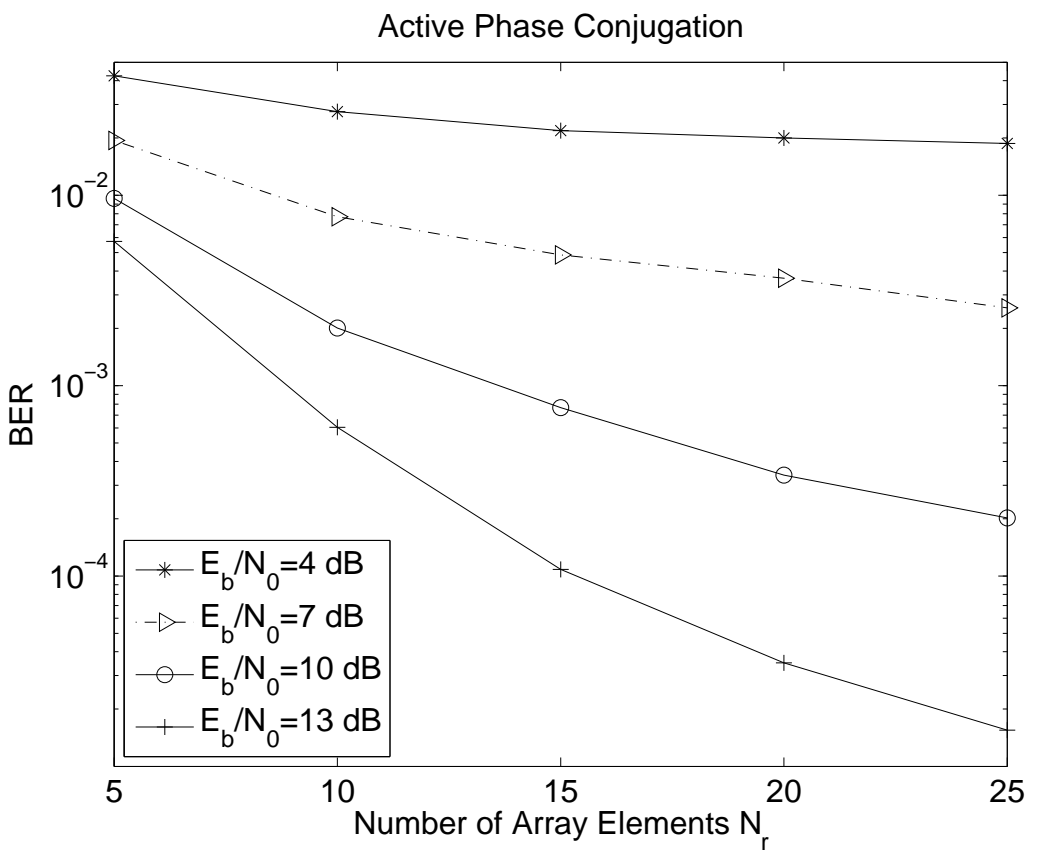

Fig. 8. Performance of APC (Scheme 5) as a function of $N_{r}$ for 11-tap WLAN channel. 


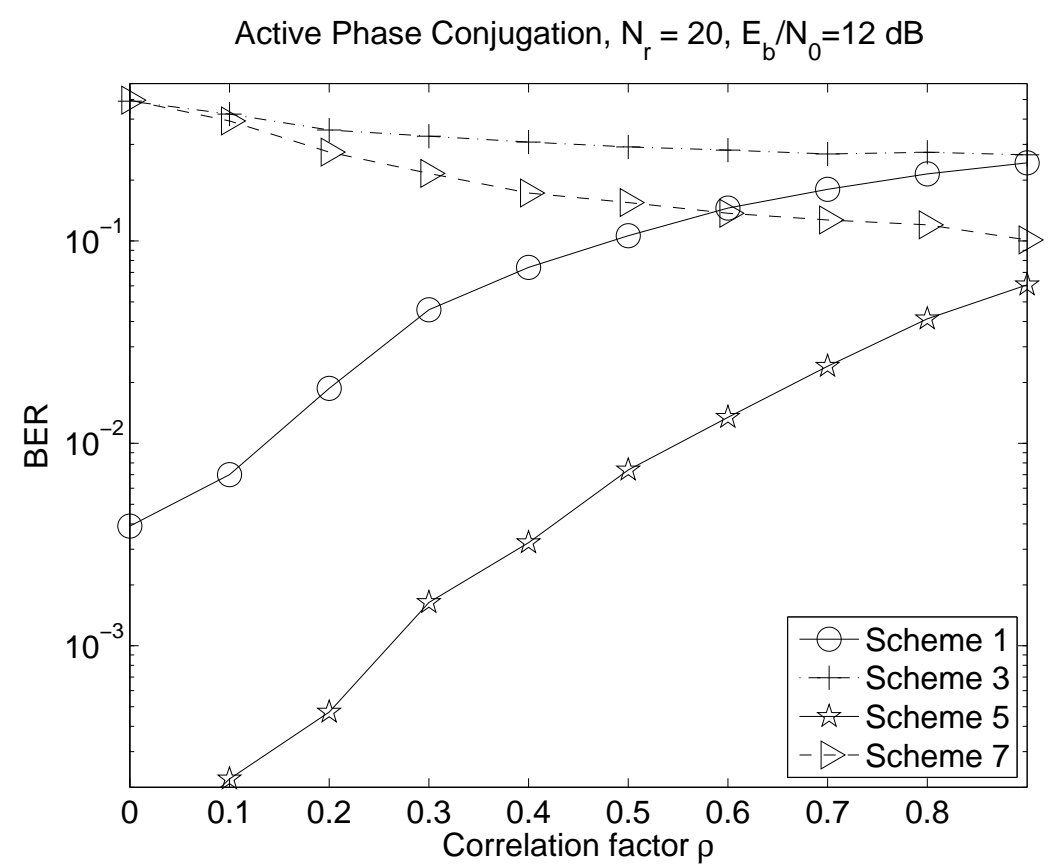

Fig. 9. Impact of antenna correlations on the performance of APC. 
TABLE I

ICF FOR 802.11 APC WLAN CHANNEL AT 2.4 GHz (ICF = 1 AT ZERO LAG).

\begin{tabular}{c|cc}
\hline \hline \multirow{2}{*}{$\begin{array}{c}\text { No. of array } \\
\text { elements }\end{array}$} & \multicolumn{2}{|c}{ max magnitude of ICF sidelobe } \\
\cline { 2 - 3 } & 11-tap channel & 51-tap channel \\
\hline 1 & 0.38 & 0.2 \\
\hline 10 & 0.07 & 0.05 \\
\hline
\end{tabular}


TABLE II

ICF FOR 802.11 APC WLAN CHANNEL AT 2.4 GHZ AS A FUNCTION OF ANTENNA CORRELATION COEFFICIENT (ICF = 1 AT ZERO LAG).

\begin{tabular}{c|c}
\hline \hline antenna correlation factor & max magnitude of ICF sidelobe \\
\hline 0.0 & 0.05 \\
\hline 0.4 & 0.25 \\
\hline 0.8 & 0.45 \\
\hline
\end{tabular}

